\title{
Dominance intensity measure within fuzzy weight oriented MAUT: An application
}

\author{
Antonio Jiménez ${ }^{\mathrm{a}, *}$, Alfonso Mateos ${ }^{\mathrm{a}}$, Pilar Sabio ${ }^{\mathrm{b}}$ \\ a Departamento de Inteligencia Artificial, Universidad Politécnica de Madrid (UPM), Campus de Montegancedo s/n, 28660 Madrid, Spain \\ ${ }^{b}$ Departamento de Economía Aplicada, Universidad Rey Juan Carlos (URJC), Facultad de Ciencias Jurídicas y Sociales, 28933 Madrid, Spain
}

A B S T R A C T

We introduce a dominance intensity measuring method to derive a ranking of alternatives to deal with incomplete information in multi-criteria decision-making problems on the basis of multi-attribute utility theory (MAUT) and fuzzy sets theory. We consider the situation where there is imprecision concerning decision-makers' preferences, and imprecise weights are represented by trapezoidal fuzzy weights. The proposed method is based on the dominance values between pairs of alternatives. These values can be computed by linear programming, as an additive multi-attribute utility model is used to rate the alternatives. Dominance values are then transformed into dominance intensity measures, used to rank the alternatives under consideration. Distances between fuzzy numbers based on the general-ization of the left and right fuzzy numbers are utilized to account for fuzzy weights.

An example concerning the selection of intervention strategies to restore an aquatic ecosystem contaminated by radionuclides illustrates the approach. Monte Carlo simulation techniques have been used to show that the proposed method performs well for different imprecision levels in terms of a hit ratio and a rank-order correlation measure.

\section{Introduction}

Most complex decision-making problems involve imprecise information [58]. It is frequently impossible to predict with certainty the alternative performances, since as they often reflect social or environmental impacts or are taken from statistics or measurements, they may be intangible.

Neither is it easy to elicit the relative importance of criteria by means of precise weights. Decision makers (DMs) may find it difficult to compare criteria or not want to reveal their preferences in public. Furthermore, in a group decision-making context, imprecision concerning preferences may be the result of a negotiation process. This situation is usually referred to as decision-making with imprecise information, with incomplete information or with partial information [49,50].

A number of papers on multi-attribute utility theory (MAUT) have dealt with incomplete information. For instance, Sage and White [54] proposed the model of imprecisely specified multiattribute utility theory (ISMAUT), where preference information about both weights and utilities is assumed not to be precise. Malakooti [38] suggested a new efficient algorithm for ranking

\footnotetext{
* Corresponding author. Tel.: +3491 3524819; fax: + 34913524819.

E-mail addresses: ajimenez@fi.upm.es (A. Jiménez), amateos@fi.upm.es (A. Mateos), pilar.sabio@urjc.es (P. Sabio).
}

alternatives when there is incomplete information about the preferences and the value of the alternatives. Ahn [1] extended Malakooti's work.

More recently, Jiménez et al. [29] accounted for missing information about some alternative performances. They proposed using the attribute range rather than redistributing the respective weights throughout the objective hierarchy.

Another possibility for dealing with imprecision within MAUT described in the literature attempts to apply the concept of pairwise and absolute dominance to eliminate inferior alternatives, leading to the so-called surrogate weighting methods $[60,4]$, and adapted classical decision rules [46,56], respectively.

Eum et al. provided linear programming characterizations of dominance and potential optimality for alternatives when information about performances and/or weights is incomplete [21]. Lee et al. extended the approach to hierarchical structures [37], and Park developed the concepts of weak potential optimality and strong potential optimality [45]. In [39], the more general case considering imprecision, described by means of fixed bounds, appears in alternative performances, as well as in weights and utilities.

More recently, different dominance measuring methods, which use information about each alternative's intensity of dominance, have been proposed $[2,41,40]$.

On the other hand, stochastic multi-criteria acceptability analysis (SMAA) is based on exploring the weight space in order to describe which scores would make each alternative the preferred 
option. Inaccurate or uncertain criteria values are represented by probability distributions and partial preference information. The $S M A A-2$ method [34] extended the original SMAA by considering all ranks in the analysis, and $S M A A-O$ [36] was designed for problems where information for some or all criteria are ordinal. Different ways of handling dependent uncertainties within SMAA2 have been analyzed in [35].

Sarabando and Dias [57] gave a brief overview of approaches proposed within the MAUT and MAVT (Multi-Attribute Value Theory) framework to deal with incomplete information.

The analytical hierarchy process $(A H P)$ is another popular method, apart from MAUT, when the information on criteria is mainly cardinal and where attributes are fully compensatory. Incomplete information within AHP has also been addressed in the literature, such as [53], where an interval of numerical values is associated with each judgment in the pairwise comparisons or $[33,25]$, dealing with missing data in AHP.

Outranking methods $[5,23,44]$ overcome the assumption that attributes are fully compensatory and there is a true ranking of alternatives just waiting to be discovered. The most widely used outranking methods are ELECTRE and PROMETHEE. ELECTRE III improved ELECTRE II to deal with inaccurate, imprecise, uncertain and ill-determined data, while PROMETHEE III is based on intervals.

Studies concerning imprecision were also conducted using the theory of fuzzy sets $[8,30]$, counting on the advances of research in arithmetic and the logical operators of fuzzy numbers, such as [62], suggesting the comparison of fuzzy numbers using a fuzzy measure of distance [17], proposing the non-additive fuzzy integral when there is dependence among criteria; or [43], introducing ordered weighted aggregation operators.

A modified fuzzy version of TOPSIS was proposed in [11], whereas [47] introduced a new specification of a fuzzy model, the fuzzy utility model which is applied for road route choice.

Preliminary works on the extension of AHP [13] to account for fuzzy numbers were proposed in $[64,6]$. A fuzzy integrated hierarchical decision-making approach was developed in [13] to solve the distribution center location selection problem. A fuzzy extension of AHP, FEAHP, is provided in [12] to deal with the selection of global suppliers, where triangular fuzzy numbers are used in the DMs' comparison judgements, whereas the final priority of the considered criteria is based on a fuzzy synthetic extent analysis [14].

Developments related to fuzzy outranking methods, such as the utilization of the PROMETHEE method with trapezoidal fuzzy numbers proposed in [24] are reviewed in [7]. More recently, an extension of the ELECTRE I method for group decision-making in a fuzzy environment was introduced in [26].

In this paper we introduce a dominance measuring method that adapts the proposal in $[41,27]$ to account for fuzzy weights, exploiting research reported in [62] on distances between fuzzy numbers based on the generalization of the left and right fuzzy numbers [19,3].

In Section 2 we review dominance-measuring methods proposed to deal with incomplete information within MAUT, which can be viewed as the groundwork of the proposed method. In Section 3 we outline the dominance-measuring method accounting for trapezoidal fuzzy weights. The approach is illustrated in Section 4 using an example concerning the selection of intervention strategies to restore an aquatic ecosystem. In Section 5, the performance of the proposed method is analyzed using Monte Carlo simulation techniques. Finally, some conclusions are discussed in Section 6.

\section{Dominance-measuring methods}

As cited in the previous section, one option described in the literature for dealing with imprecision within MAUT is to eliminate inferior alternatives based on the concept of dominance.
Let us consider a decision-making problem with $m$ alternatives, $A_{i}, i=1, \ldots, m$, and $n$ attributes, $X_{j}, j=1, \ldots, n$, where incomplete information about input parameters was incorporated into the decision-making process. $U_{i}\left(\mathbf{u}_{i}=\left(u_{i 1}, \ldots, u_{i n}\right) \in U_{i}\right)$ define the feasible region for utilities associated with alternative $A_{i}$ over each attribute. Different methods can be used to build utility functions depending on the level of knowledge and features of the attribute under consideration. When there is in-depth and precise knowledge about the attribute, the DM can directly construct a piecewise linear utility function by providing the best and the worst attribute values and some intermediate values with their respective imprecise utilities. Methods based on lotteries, such as fractile method and the extreme gambles method [22], are used when DMs have little knowledge about or are inexperienced in the domain. The GMAA decision support system, which includes the combination of two slightly modified standard procedures for utility assessment, is introduced in [28]. Incomplete information is entered as value intervals in response to the probability questions that the DM is asked, checking for consistency.

On the other hand, $W$ defines the feasible region for weights, representing the relative importance of criteria as follows:

- ordinal relations, $\mathbf{w} \in W=\left\{\mathbf{w}=\left(w_{1}, \ldots, w_{n}\right): w_{1} \geq w_{2} \geq \ldots \geq w_{n}\right\}$,

- value intervals, $\mathbf{w} \in W=\left\{\mathbf{w}=\left(w_{1}, \ldots, w_{n}\right): w_{j} \in\left[w_{j}^{l}, w_{j}^{U}\right] j=1, \ldots\right.$ $, n\}$,

- intervals for weight ratios (trade-offs), $\mathbf{w} \in W=\left\{\mathbf{w}=\left(w_{1}, \ldots\right.\right.$ ,$\left.\left.w_{n}\right): w_{j} / w_{k} \in\left[w_{j k}^{L}, w_{j k}^{U}\right], j=1, \ldots, n\right\}$,

- linear inequality constraints for weights, $\mathbf{w} \in W=\{\mathbf{w}=$ $\left.\left(w_{1}, \ldots, w_{n}\right): A w \leq c\right\}$, or

- nonlinear inequality constraints for weights, $\mathbf{w} \in W=\{\mathbf{w}=$ $\left.\left(w_{1}, \ldots, w_{n}\right): g(w) \leq 0\right\}$.

There are many weighting methods that use different questioning procedures to elicit weights, such as SWING weighting and SMARTS [20], pricing out method and TRADEOFFS weighting [31], AHP [52], or preference programming [55]. Most are adapted to account for imprecision.

We assume an additive model, which is considered a valid approximation in most real decision-making problems for the reasons described in $[48,59]$, and is widely used within MAUT,

$u\left(A_{i}\right)=\sum_{j=1}^{n} w_{j} u_{i j}=\mathbf{w}^{T} \mathbf{u}_{i}$.

Given two alternatives $A_{k}$ and $A_{b}$, the alternative $A_{k}$ dominates $A_{l}$ if $D_{k l} \geq 0, D_{k l}$ being the optimum value of the optimization problem:

$D_{k l}=\min \left\{u\left(A_{k}\right)-u\left(A_{l}\right)=\mathbf{w}^{T}\left(\mathbf{u}_{k}-\mathbf{u}_{j}\right)\right\}$

s.t. $\mathbf{u}_{k} \in U_{k}, \mathbf{u}_{j} \in U_{j}$

$\mathbf{w} \in W$.

This concept of dominance is called pairwise dominance. Another type of dominance, known as absolute dominance, can be employed [56]. Absolute dominance considers the following optimization problems:

$U_{k}=\max \left\{u\left(A_{k}\right)=\mathbf{w}^{T} \mathbf{u}_{k} \mid \mathbf{w} \in W, \mathbf{u}_{k} \in U_{k}\right\}$ and

$L_{k}=\min \left\{u\left(A_{k}\right)=\mathbf{w}^{T} \mathbf{u}_{k} \mid \mathbf{w} \in W, \mathbf{u}_{k} \in U_{k}\right\}$.

Alternative $A_{k}$ absolutely dominates $A_{l}$ if $L_{k} \geq U_{l}$, i.e., the lower bound of $A_{k}$ exceeds the upper bound of $A_{k}$. Note that if $A_{k}$ absolutely dominates $A_{l}$, then $A_{k}$ dominates $A_{l}$, but the reverse does not hold.

Note that this dominance approach often results in almost no prioritization of alternatives or too many non-dominated 
alternatives [32]. However, pairwise and absolute dominance values can be used to further prioritize competitive alternatives, and hence recommend the best alternative and fully rank alternatives. An example of how these dominance values have been employed is the adaptation of four classical decision rules to encompass an imprecise decision context [46,56].

A recent approach is to use information about each alternative's intensity of dominance, known as dominance-measuring methods. The starting point for dominance-measuring methods is the assessment of the so-called dominance matrix:

$$
D=\left(\begin{array}{ccccc}
- & D_{12} & \ldots & D_{1 m-1} & D_{1 m} \\
D_{21} & - & \ldots & D_{2 m-1} & D_{2 m} \\
D_{31} & D_{32} & - & D_{3 m-1} & D_{3 m} \\
\vdots & \vdots & \ddots & \vdots & \vdots \\
D_{m 1} & D_{m 2} & \ldots & D_{m m-1} & -
\end{array}\right),
$$

where $D_{k l}$ values are computed from expression (1).

The first two dominance-measuring methods were proposed in [2], where both dominating and dominated measures were computed from the paired dominance values, leading to a net dominance. This is used as a measure of the strength of preference in the sense that a greater net value is better.

Two new dominance-measuring methods were proposed in [41]. The first one was based on the idea first suggested by Ahn and Park. Dominating and dominated measures are computed, but they are then combined into a preference intensity rather than a net dominance measure. In the second method, alternatives were ranked on the basis of a global preference intensity measure. Paired dominance values were first transformed into preference intensities depending on the preference for alternatives $A_{k}$ and $A_{l}$. Then a global preference intensity measure was derived for each alternative $A_{k}$. This global preference intensity measure was calculated as the sum of the preference intensities of alternative $A_{k}$ compared with the other alternatives. This was used as the measure of strength of preference.

The above four methods could be applied for different representations of imprecision concerning weights. Monte Carlo simulation techniques were applied to analyze and compare their performance with other approaches, such as surrogate weighting methods [60] and [4], and adapted classical decision rules for ordinal relations regarding attribute weights, i.e., DMs ranked attributes in descending order of importance. The results showed that the preference intensity measure based method performs better than the adaptation of classical decision rules in terms of the identification of the best alternative and the overall ranking of alternatives, closely rivaling the rank-order centroid weights method, which was identified as the best approach.

Mateos et al. consider different cases with incomplete information about weights [40], specifically, weight intervals, weights fitting independent normal probability distributions or weights represented by triangular or trapezoidal fuzzy numbers. Monte Carlo simulation is again used to compare dominance-measuring methods with SMAA and SMAA-2 methods where intervals account for imprecision in weights.

The dominance concept has also been used within fuzzy multicriteria analysis. Although the most widely used definition is based on the maximum degree of membership of the two considered fuzzy numbers (see, e.g. $[16,15]$ ), other definitions have been proposed, such as the comparison of overlapped and non-overlapped areas among the two fuzzy numbers [61], or their arithmetic difference [67]. In [66], Yeh and Deng combine arithmetic difference with the notion of fuzzy reference set to address the fuzzy ranking problem.

The dominance-measuring method that we introduce in this paper adapts the proposal in $[41,27]$ to account for fuzzy weights, exploiting research reported in [62] on distances between fuzzy numbers based on the generalization of the left and right fuzzy numbers $[19,3]$.

\section{A dominance-measuring method accounting for trapezoidal fuzzy weights}

First, let us review several concepts on fuzzy sets that will be used in the proposed method. A fuzzy set $\tilde{a}=\left(a_{1}, a_{2}, a_{3}, a_{4}\right)$ is called a generalization of the left and right fuzzy numbers (GLRFN) when its membership function is defined as

$\mu_{\tilde{a}}(x)= \begin{cases}L\left(\frac{a_{2}-x}{a_{2}-a_{1}}\right) & \text { if } a_{1} \leq x \leq a_{2} \\ 1 & \text { if } a_{2} \leq x \leq a_{3} \\ R\left(\frac{x-a_{3}}{a_{4}-a_{3}}\right) & \text { if } a_{3} \leq x \leq a_{4} \\ 0 & \text { otherwise, }\end{cases}$

where $L$ and $R$ are strictly decreasing functions defined in $[0,1]$ and satisfying the conditions:

$L(x)=R(x)=1 \quad$ if $x \leq 0$,
$L(x)=R(x)=0 \quad$ if $x \geq 0$.

For $a_{2}=a_{3}$, we have the classical Dubois-Prade definition of right and left triangular fuzzy numbers [19]. Trapezoidal fuzzy numbers are a special case of GLRFN with $L(x)=R(x)=1-x$. A GLRFN is denoted as $\tilde{a}=\left(a_{1}, a_{2}, a_{3}, a_{4}\right)_{L \tilde{a}-R \tilde{a}}$ and a $\alpha$-cut of $\tilde{a}$ is defined as

$\tilde{a}(\alpha)=\left(\tilde{a}_{L}(\alpha), \tilde{a}_{R}(\alpha)\right)=\left(a_{2}-\left(a_{2}-a_{1}\right) a_{3} L_{\hat{a}}^{-1}(\alpha), a_{3}-\left(a_{4}-a_{3}\right) a_{3} R_{\tilde{a}}^{-1}(\alpha)\right)$.

Tran and Duckstein [62] define the distance between two GLFRN fuzzy numbers $\tilde{a}$ and $\tilde{b}$ as

$$
\begin{aligned}
& D^{2}(\tilde{a}, \tilde{b}, f) \\
& =\left\{\int_{0}^{1}\left\{\begin{array}{c}
{\left[\frac{\tilde{a}_{L}(\alpha)+\tilde{a}_{R}(\alpha)}{2}-\frac{\tilde{b}_{L}(\alpha)+\tilde{b}_{R}(\alpha)}{2}\right]^{2}+} \\
\left.+\frac{1}{3}\left[\left(\frac{\tilde{a}_{L}(\alpha)+\tilde{a}_{R}(\alpha)}{2}\right)^{2}+\frac{\tilde{b}_{L}(\alpha)+\tilde{b}_{R}(\alpha)}{2}\right)^{2}\right]
\end{array}\right\}\right. \\
& \quad \times f(\alpha) d \alpha\} / \int f(\alpha) d \alpha .
\end{aligned}
$$

The function $f(\alpha)$, which serves as a weight function, is positive continuous in $[0,1]$, the distance being computed as a weighted sum of distances between two intervals across all the $\alpha$-cuts from 0 to 1 . DM participation is flexible thanks to the presence of function $f$. For example, when the DM is risk-neutral, $f(\alpha)=\alpha$ seems to be reasonable. A risk-averse DM might want to attach more weight to information at a higher $\alpha$ level by using other functions, such as $f(\alpha)=\alpha^{2}$ or a higher power of $\alpha$. A constant $(f(\alpha)=1)$, or even a decreasing function $f$, can be utilized for a risk-prone DM.

For the particular case of the distance of a trapezoidal fuzzy number $\tilde{a}=\left(a_{1}, a_{2}, a_{3}, a_{4}\right)$ to a constant (specifically 0$)$, we have

$$
\begin{aligned}
& \text { 1. If } f(\alpha)=\alpha \text {, then } \\
& \qquad \begin{aligned}
D^{2}(\tilde{a}, 0, f)= & \left(\frac{a_{2}+a_{3}}{2}\right)^{2}+\frac{1}{3}\left(\frac{a_{2}+a_{3}}{2}\right)\left[\left(a_{4}-a_{3}\right)-\left(a_{2}-a_{1}\right)\right] \\
& +\frac{2}{3}\left(\frac{a_{3}-a_{2}}{2}\right)^{2}+\frac{1}{9}\left(\frac{a_{3}-a_{2}}{2}\right)\left[\left(a_{4}-a_{3}\right)+\left(a_{2}-a_{1}\right)\right]
\end{aligned}
\end{aligned}
$$




$$
+\frac{1}{18}\left[\left(a_{4}-a_{3}\right)^{2}+\left(a_{2}-a_{1}\right)^{2}\right]-\frac{1}{18}\left[\left(a_{2}-a_{1}\right)\left(a_{4}-a_{3}\right)\right] .
$$

2. If $f(\alpha)=1$, then

$$
\begin{aligned}
D^{2}(\tilde{a}, 0, f)= & \left(\frac{a_{2}+a_{3}}{2}\right)^{2}+\frac{1}{2}\left(\frac{a_{2}+a_{3}}{2}\right)\left[\left(a_{4}-a_{3}\right)-\left(a_{2}-a_{1}\right)\right]+\frac{1}{3}\left(\frac{a_{3}-a_{2}}{2}\right) \\
& +\frac{1}{6}\left(\frac{a_{3}-a_{2}}{2}\right)\left[\left(a_{4}-a_{3}\right)+\left(a_{2}-a_{1}\right)\right]+\frac{1}{9}\left[\left(a_{4}-a_{3}\right)^{2}+\left(a_{2}-a_{1}\right)^{2}\right] \\
& -\frac{1}{9}\left[\left(a_{2}-a_{1}\right)\left(a_{4}-a_{3}\right)\right] .
\end{aligned}
$$

3. If $f(\alpha)=\alpha^{2}$, then

$$
\begin{aligned}
D^{2}(\tilde{a}, 0, f)= & \left(\frac{a_{2}+a_{3}}{2}\right)^{2}+\frac{1}{4}\left(\frac{a_{2}+a_{3}}{2}\right)\left[\left(a_{4}-a_{3}\right)-\left(a_{2}-a_{1}\right)\right] \\
& +\left(\frac{a_{3}-a_{2}}{2}\right)^{2}+\frac{1}{12}\left(\frac{a_{3}-a_{2}}{2}\right)\left[\left(a_{4}-a_{3}\right)+\left(a_{2}-a_{1}\right)\right] \\
& -\frac{1}{144}\left[\left(a_{4}-a_{3}\right)^{2}+\left(a_{2}-a_{1}\right)^{2}\right]+\frac{1}{96}\left[\left(a_{2}-a_{1}\right)\left(a_{4}-a_{3}\right)\right] .
\end{aligned}
$$

Let us now introduce the new dominance-measuring method. We now consider that imprecise weights are represented by trapezoidal fuzzy numbers $\tilde{w}_{j}, j=1, \ldots, n$. Different direct and indirect methods for constructing fuzzy sets have been proposed for one and multiple experts in the literature [18]. In $[9,10]$, the advantages, disadvantages and limitations of four elicitation methods are discussed, whereas [63] reviews three basic views of the representation of membership functions, together with fundamental measurements of linguistic terms of linguistic variables.

Then, the optimization problem (1) can be now represented by

$$
\begin{aligned}
& \tilde{D}_{k l}=\min \left\{\sum_{j=1}^{n} \tilde{w}_{j}\left(\mathbf{u}_{k}-\mathbf{u}_{l}\right)=\sum_{j=1}^{n}\left(w_{j 1}, w_{j 2}, w_{j 3}, w_{j 4}\right)\left(\mathbf{u}_{k}-\mathbf{u}_{l}\right)\right. \\
& \left.=\left(D_{k l 1}, D_{k l 2}, D_{k l 3}, D_{k l 4}\right)\right\} \\
& \text { s.t. } \quad \mathbf{u}_{k} \in U_{k}, \mathbf{u}_{l} \in U_{l} \\
& \quad \tilde{\mathbf{w}} \in W,
\end{aligned}
$$

where $\tilde{\mathbf{w}} \in W=\left\{\tilde{\mathbf{w}}=\left(\tilde{w}_{1}, \ldots, \tilde{w}_{n}\right): \tilde{w}_{j} j=1, \ldots, n,\right\}$, being $\tilde{w}_{j}$ trapezoidal fuzzy numbers.

Then, the first step in the proposed method is to compute the above trapezoidal fuzzy numbers, see Fig. 1. Consequently, each element in the dominance matrix is a trapezoidal fuzzy number:

$$
D=\left(\begin{array}{ccccc}
- & \tilde{D}_{12} & \ldots & \tilde{D}_{1 m-1} & \tilde{D}_{1 m} \\
\tilde{D}_{21} & - & \ldots & \tilde{D}_{2 m-1} & \tilde{D}_{2 m} \\
\tilde{D}_{31} & \tilde{D}_{32} & - & \tilde{D}_{3 m-1} & \tilde{D}_{3 m} \\
\vdots & \vdots & \ddots & \vdots & \vdots \\
\tilde{D}_{m 1} & \tilde{D}_{m 2} & \ldots & \tilde{D}_{m m-1} & -
\end{array}\right) .
$$

Next, we compute the strength of dominance of alternative $A_{k}$ by adding the trapezoidal fuzzy numbers in the $k$ th row of $D$

$$
\tilde{d}_{k}=\left(d_{k 1}, d_{k 2}, d_{k 3}, d_{k 4}\right)=\sum_{\substack{i=1 \\ i \neq k}}^{m} \tilde{D}_{k l}=\left(\sum_{\substack{i=1 \\ i \neq k}}^{m} D_{k l 1}, \sum_{\substack{i=1 \\ i \neq k}}^{m} D_{k 12}, \sum_{\substack{i=1 \\ i \neq k}}^{m} D_{k 13}, \sum_{\substack{i=1 \\ i \neq k}}^{m} D_{k \mid 4}\right) \text {. }
$$

Finally, a dominance intensity, $D I_{k}$, for each alternative $A_{k}$ is computed as the proportion of the positive part of the fuzzy number $\tilde{d}_{k}$ by the distance of the fuzzy number to zero. Specifically, the dominance intensity for alternative $A_{k}$ is computed according to the location of $\tilde{d}_{k}$ as follows:

1. If $\tilde{d}_{k}$ is completely located at the left of zero, then $D I_{k}$ is minus the distance of $\tilde{d}_{k}$ to zero, because there is no positive part in $\tilde{d}_{k}$.

2. If $\tilde{d}_{k}$ is completely located at the right of zero, then $D I_{k}$ is the distance of $\tilde{d}_{k}$ to zero, because there is no negative part existing in $\tilde{d}_{k}$.

3. If $\tilde{d}_{k}$ includes the zero in its base, then the fuzzy number will have a part on the right of zero that we denote $\tilde{d}_{k}^{R}$ and another part on the left of zero that we denote $\tilde{d}_{k}^{L}$. $D I_{k}$ is the proportion that represents $\tilde{d}_{k}^{R}$ with respect to $\tilde{d}_{k}$ by the distance of $\tilde{d}_{k}$ to zero less the proportion that represents $\tilde{d}_{k}^{R}$ with respect to $\tilde{d}_{k}$ by the distance of $\tilde{d}_{k}$ to zero.

Next, we analyze each one of these cases in more detail:

- If $d_{k 4}<0$, see Fig. $2(a)$, then the dominance intensity of alternative $A_{k}$ is defined as $D I_{k}=-D\left(\tilde{d}_{k}, 0, f\right)$.

- If $d_{k 1}>0$, see Fig. 2(b), then the dominance intensity of alternative $A_{k}$ is defined as $D I_{k}=D\left(\tilde{d}_{k}, 0, f\right)$.

- If $d_{k 1}<0$ and $d_{k 2}>0$, see Fig. 2(c), the corresponding trapezoidal fuzzy number is divided by the vertical axis (at zero) into two parts. The left part $\tilde{d}_{k}^{L}$ represents the proportion:

$$
\frac{\frac{-d_{k 1} \frac{-d_{k 1}}{d_{k 2}-d_{k 1}}}{2}}{\frac{d_{k 4}+d_{k 3}-d_{k 2}-d_{k 1}}{2}}=\frac{\left(d_{k 1}\right)^{2}}{\left(d_{k 4}+d_{k 3}-d_{k 2}-d_{k 1}\right)\left(d_{k 2}-d_{k 1}\right)} \text {, }
$$

whereas the right part $\tilde{d}_{k}^{R}$ represents the proportion

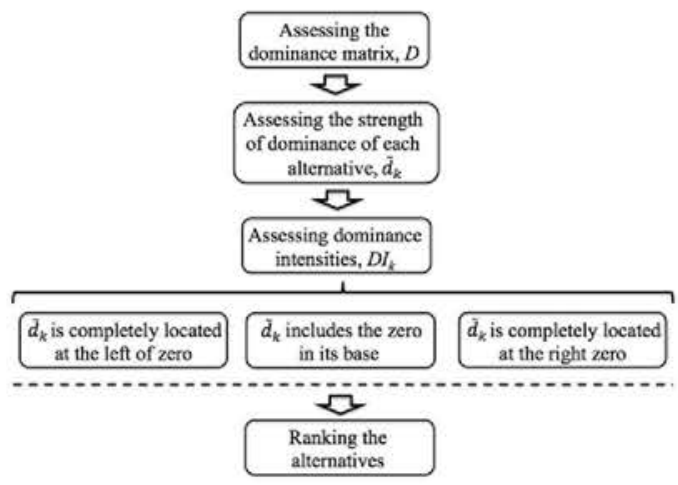

Fig. 1. Flowchart of the proposed method. $\frac{\frac{d_{k 4}+d_{k 3}-d_{k 2}-d_{k 1}}{2}-\frac{\left(-d_{k 1}\right)\left(-d_{k 1}\right)}{2\left(d_{k 2}-d_{k 1}\right)}}{\frac{d_{k 4}+d_{k 3}-d_{k 2}-d_{k 1}}{2}}=\frac{d_{k 2}\left(-d_{k 2}+d_{k 3}+d_{k 4}\right)-d_{k 1}\left(d_{k 3}+d_{k 4}\right)}{\left(d_{k 2}-d_{k 1}\right)\left(d_{k 4}+d_{k 3}-d_{k 2}-d_{k 1}\right)}$

The dominance intensity of alternative $A_{k}$ is defined as

$$
\begin{aligned}
D I_{k}= & \frac{d_{k 2}\left(-d_{k 2}+d_{k 3}+d_{k 4}\right)-d_{k 1}\left(d_{k 3}+d_{k 4}\right)}{\left(d_{k 2}-d_{k 1}\right)\left(d_{k 4}+d_{k 3}-d_{k 2}-d_{k 1}\right)} D\left(\tilde{d}_{k}, 0, f\right) \\
& -\frac{\left(d_{k 1}\right)^{2}}{\left(d_{k 4}+d_{k 3}-d_{k 2}-d_{k 1}\right)\left(d_{k 2}-d_{k 1}\right)} D\left(\tilde{d}_{k}, 0, f\right) .
\end{aligned}
$$

- If $d_{k 3}<0$ and $d_{k 4}>0$, Fig. 2(d), the corresponding trapezoidal fuzzy number is again divided by the vertical axis into two 


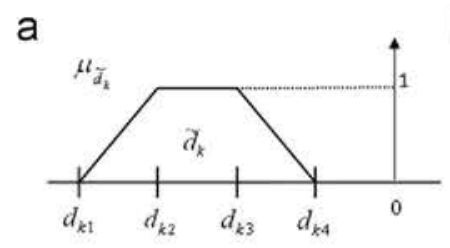

d

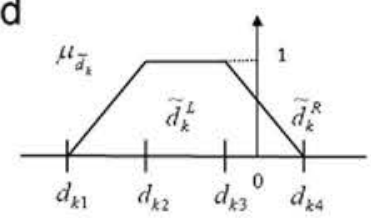

b

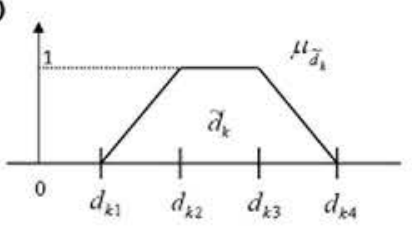

e

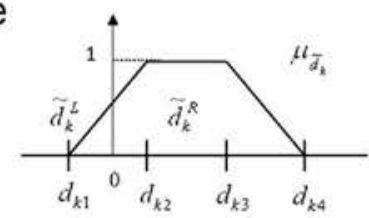

C

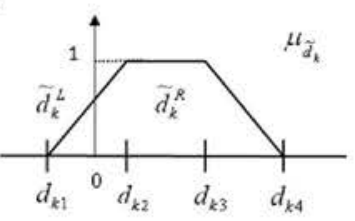

f

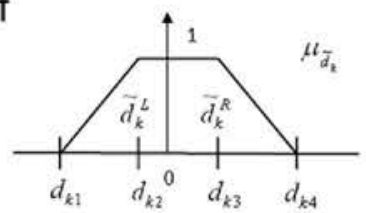

Fig. 2. Possible locations of $\tilde{d}_{k}$.

parts, $\tilde{d}_{k}^{L}$ and $\tilde{d}_{k}^{R}$, represented by the proportions

$$
\begin{gathered}
\frac{\frac{d_{k 4}+d_{k 3}-d_{k 2}-d_{k 1}}{2}-\frac{d_{k 4} \frac{d_{k 4}}{d_{k 4}-d_{k 3}}}{2}}{\frac{d_{k 4}+d_{k 3}-d_{k 2}-d_{k 1}}{2}} \\
=\frac{d_{k 4}\left(d_{k 4}-d_{k 2}-d_{k 1}\right)-d_{k 3}\left(d_{k 3}-d_{k 2}-d_{k 1}\right)}{\left(d_{k 4}+d_{k 3}-d_{k 2}-d_{k 1}\right)\left(d_{k 4}-d_{k 3}\right)}
\end{gathered}
$$

and

$$
\frac{\frac{d_{k 4} \frac{d_{k 4}}{d_{k 4}-d_{k 3}}}{2}}{\frac{d_{k 4}+d_{k 3}-d_{k 2}-d_{k 1}}{2}}=\frac{\left(d_{k 4}\right)^{2}}{\left(d_{k 4}-d_{k 3}\right)\left(d_{k 4}+d_{k 3}-d_{k 2}-d_{k 1}\right)},
$$

respectively, and dominance intensity of alternative $A_{k}$ is

$$
\begin{aligned}
D I_{k}= & \frac{\left(d_{k 4}\right)^{2}}{\left(d_{k 4}-d_{k 3}\right)\left(d_{k 4}+d_{k 3}-d_{k 2}-d_{k 1}\right)} D\left(\tilde{d}_{k}, 0, f\right) \\
& -\frac{d_{k 4}\left(d_{k 4}-d_{k 2}-d_{k 1}\right)-d_{k 3}\left(d_{k 3}-d_{k 2}-d_{k 1}\right)}{\left(d_{k 4}+d_{k 3}-d_{k 2}-d_{k 1}\right)\left(d_{k 4}-d_{k 3}\right)} D\left(\tilde{d}_{k}, 0, f\right) .
\end{aligned}
$$

- If $d_{k 2}<0$ and $d_{k 3}>0$, see Fig. $2(\mathrm{e}), \tilde{d}_{k}^{L}$ and $\tilde{d}_{k}^{R}$ are

$$
\frac{\frac{d_{k 4}-d_{k 3}}{2}+d_{k 3}}{\frac{d_{k 4}+d_{k 3}-d_{k 2}-d_{k 1}}{2}}=\frac{d_{k 4}+d_{k 3}}{d_{k 4}+d_{k 3}-d_{k 2}-d_{k 1}} \text {. }
$$

and

$$
\frac{\frac{d_{k 2}-d_{k 1}-d_{k 2}}{2}}{\frac{d_{k 4}+d_{k 3}-d_{k 2}-d_{k 1}}{2}}=\frac{-d_{k 2}-d_{k 1}}{d_{k 4}+d_{k 3}-d_{k 2}-d_{k 1}},
$$

respectively, and the dominance intensity of alternative $A_{k}$ is

$$
D I_{k}=\frac{-d_{k 2}-d_{k 1}}{d_{k 4}+d_{k 3}-d_{k 2}-d_{k 1}} D\left(\tilde{d}_{k}, 0, f\right)-\frac{d_{k 4}+d_{k 3}}{d_{k 4}+d_{k 3}-d_{k 2}-d_{k 1}} D\left(\tilde{d}_{k}, 0, f\right) .
$$

Once the dominance intensity has been computed for each alternative $A_{k}, D I_{k}$, the alternatives are ranked accordingly, where the best (rank 1 ) is the alternative with greatest $D I_{k}$ and the worst is the alternative with the least $D I_{k}$.

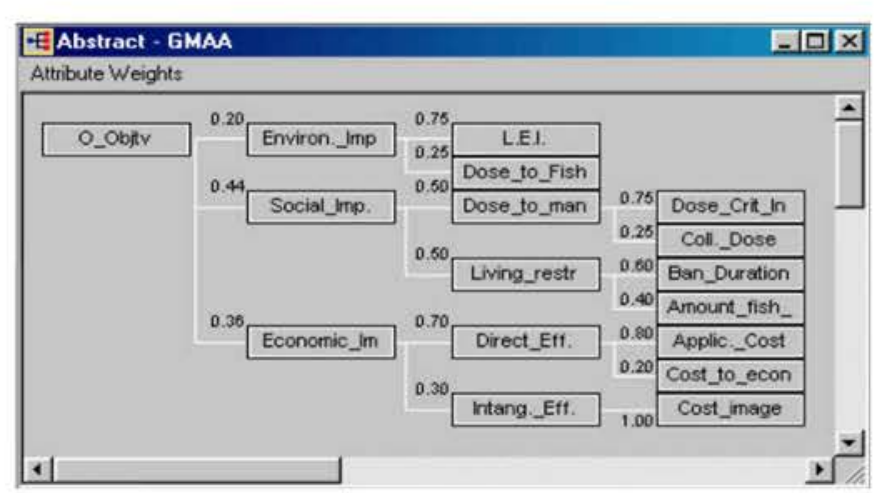

Fig. 3. Objective hierarchy for lake Svyatoye.

\section{Application to the restoration of aquatic ecosystems}

In this section we illustrate the application of the proposed method to the evaluation of intervention strategies for restoring a radionuclide contaminated aquatic ecosystem, lake Svyatoye, which was heavily contaminated after the Chernobyl accident [51]. This is a complex decision-making problem simultaneously accounting for environmental, social and economic impact.

Fig. 3 shows the objective hierarchy representing all the relevant aspects to be considered in the evaluation of the intervention strategies.

Environmental Impact (Environ._Imp) is one of the main objectives of the decision analysis. It was divided into Lake Ecosystem Index (LEI), a simple and rational approach for measuring the ecological status of a lake and Radiation Dose to Biota (Dose_to_Fish). Social Impact (Social_Imp.) was handled by two sub-objectives: Minimizing Impact on Health (Dose_to_Man) and Living Restrictions (Living_restr).

Regarding dose to man, we focused on the Dose to Critical Individuals (Dose Crit In), who should never receive radiation levels above thresholds for early health effects, and Collective Dose (Coll._Dose), which was linearly related to the increase in the risk of developing serious latent effects, mainly cancers. As regards living restrictions, other impacts were taken into consideration. These include countermeasures affecting the direct consumption of fish for food or its processing in the food industry, drinking water and water used by the food industry, the use of water for crops irrigation and the recreational uses of water bodies. For all these objectives, the attributes were the Amount of Fish Affected by restrictions (Amount_fish), as well as the duration of such restrictions (Ban_Duration).

Finally, Economic Impact (Economic_Im) was divided into Direct Effects (Direct_Eff.), more amenable to quantification, and 
Intangible Effects (Intang._Eff.), like loss-of-image and adverse market reactions for the concerned area, which could also be subjectively rated by the user. The direct effects include the costs generated by the different bans on or restrictions to normal living conditions, which can be sub-divided into Costs to the Economy (Cost_to_econ) and the more subjective costs of lost recreation, and Application Costs (Applic._Cost), i.e., costs of chemical and physical remedial countermeasures.

Alternative performances were described under uncertainty by uniformly distributed intervals. On the other hand, imprecise component utilities were assessed, leading to classes of utility functions. Fig. 4 shows the corresponding weights, i.e., the indicators of the influence of the individual decision-making criteria. For more information about the problem, component utility and weight elicitation and the resulting ranking of alternatives, see [51].

The intervention strategies considered for evaluation are shown in Table 1, including the combination of chemical countermeasures with fishing bans so as to reduce the radiological and environmental impact.

The original imprecise weights elicited by DMs are shown in Fig. 4. The possibility of using trapezoidal fuzzy numbers rather than intervals was not considered at that time. However, DMs agreed at later meetings that trapezoidal fuzzy numbers would better reflect their preferences. As a starting point, weight intervals were transformed into trapezoidal fuzzy numbers, $\tilde{w}_{j}=\left(w_{j 1}, w_{j 2}, w_{j 3}, w_{j 4}\right)$, as follows. Given an imprecise weight $\left[w_{j}^{L}, w_{j}^{U}\right]$, the base of the trapezoid is an interval such as $w_{j 1}=w_{j}^{L}$ and $w_{j 4}=w_{j}^{U}$, whereas the length of the lower base is a third part of the higher base, i.e., $w_{j 2}=w_{j}^{L}+\left(w_{j}^{U}-w_{j}^{L}\right) / 3$ and $w_{j 3}=w_{j}^{U}-$ $\left(w_{j}^{U}-w_{j}^{L}\right) / 3$.

As performance intervals and classes of component utility functions are considered, the resolution of optimization problem (2) is equivalent to solving [42]:

$\tilde{D}_{k l}=u\left(A_{k}\right)-u\left(A_{l}\right)=\tilde{\mathbf{w}}^{T} \mathbf{z}_{k l}^{*}$,

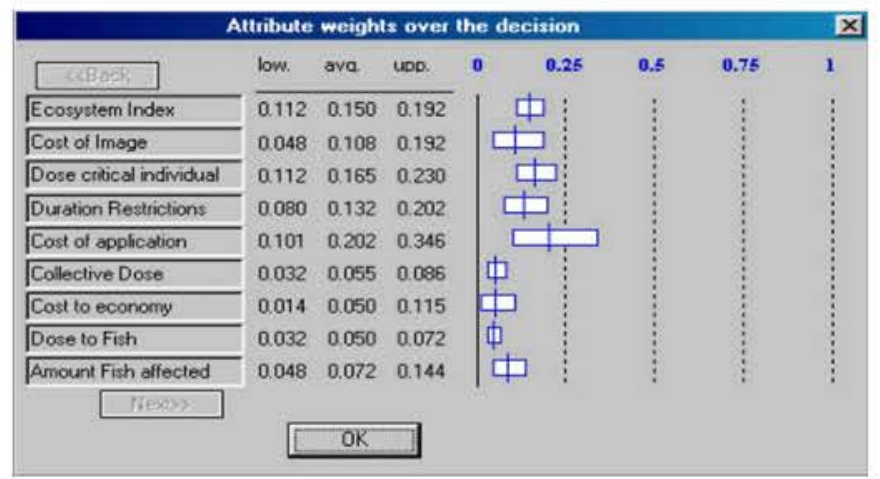

Fig. 4. Imprecise attribute weights. where $\tilde{\mathbf{w}} \in W, \mathbf{z}_{k l}^{*}=\left(z_{k l 1}^{*}, \ldots, z_{k l n}^{*}\right)$ and $z_{k l j}^{*}$ is the optimum value of the following optimization problem:

$$
\begin{aligned}
\min \quad & z_{k l j}=u_{j}\left(x_{k j}\right)-u_{j}\left(x_{l j}\right) \\
\text { s.t. } & x_{k j}^{L} \leq x_{k j} \leq x_{k j}^{U}, \quad j=1, \ldots, n \\
& x_{l j}^{L} \leq x_{l j} \leq x_{l j}^{U}, \quad j=1, \ldots, n \\
& u_{j}^{L}\left(x_{k j}\right) \leq u_{j}\left(x_{k j}\right) \leq u_{j}^{U}\left(x_{k j}\right), \quad j=1, \ldots, n \\
& u_{j}^{L}\left(x_{l j}\right) \leq u_{j}\left(x_{l j}\right) \leq u_{j}^{U}\left(x_{l j}\right), \quad j=1, \ldots, n .
\end{aligned}
$$

The optimal solution of problem (3) can be determined very simply for certain types of utility functions [42]. Specifically, if the utility function is monotonically increasing or decreasing, then $z_{k l j}^{*}=u_{j}^{L}\left(x_{k j}^{L}\right)-u_{j}^{U}\left(x_{l j}^{U}\right)$ or $z_{k l j}^{*}=u_{j}^{U}\left(x_{k j}^{U}\right)-u_{j}^{L}\left(x_{l j}^{L}\right)$, respectively.

The respective optimization problems were solved to compute the dominance matrix, and the strengths of dominance for each alternative are

$\tilde{d}_{1}=(-0.1438,0.1334,0.4118,0.7174)$,

$\tilde{d}_{2}=(0.7752,1.0954,1.4157,1.7712)$,

$\tilde{d}_{3}=(0.2493,0.4764,0.7035,0.9730)$,

$\tilde{d}_{4}=(-0.1579,0.3522,0.8623,1.3958)$,

$\tilde{d}_{5}=(0.2725,0.5441,0.8158,1.1096)$,

$\tilde{d}_{6}=(-0.7043,-0.3217,0.0609,0.4724)$

$\tilde{d}_{7}=(0.4777,0.7281,0.9782,1.2489)$.

Finally, dominance intensities, $D I_{k}$, are computed for each alternative $A_{k}$ for the case of a risk-prone, neutral and risk-averse DM to obtain the corresponding ranking of alternatives, see Table 2. Table 2 also shows the ranking for the original problem in [51].

The three alternative rankings match the original ranking except for alternatives $A_{5}$ (Lake liming) and $A_{7}$ (Wetland liming), which switch positions in the ranking (second and third). This switch can be justified taking into account that the average overall utilities for both intervention strategies on which the ranking is based, are almost the same. Moreover, the upper overall utility for $A_{7}$ is greater than for $A_{5}$ [51].

To illustrate the assessment of dominance intensities listed in Table 2, we consider the case of the risk-prone $D M$ and $A_{1}$ (No action) alternative. As $d_{11}=-0.1438<0$ and $d_{12}=0.1334>0$, then

$$
\begin{aligned}
& \tilde{d}_{1}^{L}=\frac{\left(d_{11}\right)^{2}}{\left(d_{14}+d_{13}-d_{12}-d_{11}\right)\left(d_{k 2}-d_{k 1}\right)}=0.06534, \\
& \tilde{d}_{1}^{R}=\frac{d_{12}\left(-d_{12}+d_{13}+d_{14}\right)-d_{11}\left(d_{13}+d_{14}\right)}{\left(d_{12}-d_{11}\right)\left(d_{14}+d_{13}-d_{12}-d_{11}\right)}=0.9346,
\end{aligned}
$$

Table 1

Intervention strategies.

\begin{tabular}{ll}
\hline Intervention strategies & Description \\
\hline$A_{1}:$ No action & Natural evolution of the situation without intervention \\
$A_{2}:$ Potash & 15 tonnes of potassium chloride added to the lake in April 1987 \\
$A_{3}:$ Fertilizer & $800 \mathrm{~kg}$ of fertilizer added to the lake between April and July $1987,1988,1989,1990, \mathrm{i}, \mathrm{e}, 200 \mathrm{~kg} / \mathrm{month}$ \\
$A_{4}:$ Food ban & Automatic fish consumption ban when ${ }^{137} \mathrm{Cs}$ content in fish is greater than $1000 \mathrm{~Bq} / \mathrm{kg}$ \\
$A_{5}:$ Lake liming & 15 tonnes of lime added to the lake in April 1987 \\
$A_{6}:$ Sediment removal & $250,000 \mathrm{~m}_{2}$ of sediments removed from the lake down to a depth of $10 \mathrm{~cm}$ from May to June 1990 \\
$A_{7}:$ Wetland liming & 30 ton of lime added to the catchment in May 1987 \\
\hline
\end{tabular}


Table 2

Dominance intensities and ranking of alternatives.

\begin{tabular}{|c|c|c|c|c|c|c|c|}
\hline \multirow{2}{*}{$\begin{array}{l}\text { Intervention } \\
\text { strategies }\end{array}$} & \multirow{2}{*}{$\begin{array}{l}\text { Original } \\
\text { ranking }\end{array}$} & \multicolumn{2}{|c|}{ Risk prone } & \multicolumn{2}{|l|}{ Neutral } & \multicolumn{2}{|c|}{ Risk averse } \\
\hline & & $D I_{k}$ & Ranking & $D I_{k}$ & Ranking & $D I_{k}$ & Ranking \\
\hline$A_{1}$ & 6 & 0.2853 & 6 & 0.2798 & 6 & 0.2850 & 6 \\
\hline$A_{2}$ & 1 & 1.2798 & 1 & 1.2754 & 1 & 1.2763 & 1 \\
\hline$A_{3}$ & 5 & 0.6174 & 5 & 0.6122 & 5 & 0.6129 & 5 \\
\hline$A_{4}$ & 4 & 0.6546 & 4 & 0.6472 & 4 & 0.6571 & 4 \\
\hline$A_{5}$ & 2 & 0.7055 & 3 & 0.7019 & 3 & 0.7041 & 3 \\
\hline$A_{6}$ & 7 & 0.0837 & 7 & 0.0811 & 7 & 0.0864 & 7 \\
\hline$A_{7}$ & 3 & 0.8719 & 2 & 0.8689 & 2 & 0.8702 & 2 \\
\hline
\end{tabular}

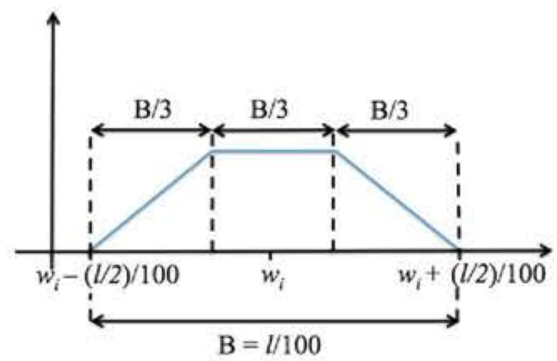

Fig. 5. Trapezoidal fuzzy number construction.

and

$$
\begin{aligned}
D I_{1}= & \tilde{d}_{1}^{R} \times D\left(\tilde{d}_{1}, 0, f\right)-\tilde{d}_{1}^{L} \times D\left(\tilde{d}_{1}, 0, f\right)=0.9346 \times 0.1077-0.06534 \\
& \times 0.1077=0.2853 .
\end{aligned}
$$

\section{Performance analysis based on monte carlo simulation techniques}

The performance of the proposed dominance measuring method accounting for fuzzy weights has been analyzed on the basis of Monte Carlo simulation techniques using two measures of efficacy: hit ratio and rank-order correlation $[2,39]$. The hit ratio is the proportion of all cases in which the method selects the same best alternative as in the TRUE ranking. Rank-order correlation represents how similar the overall structures ranking alternatives are in the TRUE ranking and in the ranking derived from the proposed method. It is calculated using Kendall's $\tau$ [65]: $\tau=1-2 \times$ (number of pairwise preference violations)/(total number of pair preferences).

Four different levels of alternatives $(m=3,5,7,10)$ and five different levels of attributes $(n=3,5,7,10,15)$ were considered in order to validate the results. Also, 20000 trials were performed for each of the 20 design elements. For simulation purpose, different routines were implemented in Python v3.1.3, which provides the Mersenne Twister method pseudo-random number generator. It is one of the most extensively tested random number generators in existence and produces 53-bit precision floats and has a period of $2^{19937}-1$.

First, component utilities for each alternative in each attribute are randomly generated from a uniform distribution in $(0,1)$, leading to an $m \times n$ matrix. The columns in this matrix are normalized to make the smallest value 0 and the largest 1 , and dominated alternatives are removed.

Next, attribute weights representing the relative attribute importance are generated. These weights are the TRUE weights and the derived ranking of alternatives will be denoted as the TRUE ranking. Those weights are then transformed into trapezoidal fuzzy numbers
Table 3

\begin{tabular}{|c|c|c|c|c|c|c|c|}
\hline \multirow[t]{2}{*}{ Alternatives } & \multirow[t]{2}{*}{ Attributes } & \multicolumn{3}{|c|}{ Hit ratio } & \multicolumn{3}{|c|}{ Kendall's $\tau$} \\
\hline & & $\begin{array}{l}\text { Risk } \\
\text { prone }\end{array}$ & Neutral & $\begin{array}{l}\text { Risk } \\
\text { averse }\end{array}$ & $\begin{array}{l}\text { Risk } \\
\text { prone }\end{array}$ & Neutral & $\begin{array}{l}\text { Risk } \\
\text { averse }\end{array}$ \\
\hline 3 & 3 & 99.67 & 99.67 & 99.68 & 99.60 & 99.61 & 99.61 \\
\hline 3 & 5 & 99.19 & 99.22 & 99.25 & 99.02 & 99.06 & 99.07 \\
\hline 3 & 7 & 98.39 & 98.40 & 98.43 & 98.05 & 98.07 & 98.10 \\
\hline 3 & 10 & 96.34 & 96.35 & 96.37 & 95.92 & 95.96 & 95.97 \\
\hline 3 & 15 & 90.69 & 90.70 & 90.76 & 89.65 & 89.70 & 89.73 \\
\hline 5 & 3 & 99.19 & 99.19 & 99.21 & 99.20 & 99.21 & 99.21 \\
\hline 5 & 5 & 98.80 & 98.84 & 98.85 & 98.48 & 98.51 & 98.51 \\
\hline 5 & 7 & 98.33 & 98.39 & 98.38 & 97.22 & 97.25 & 97.25 \\
\hline 5 & 10 & 97.44 & 97.53 & 97.59 & 94.03 & 94.07 & 94.07 \\
\hline 5 & 15 & 95.68 & 95.84 & 95.91 & 83.83 & 83.90 & 83.92 \\
\hline 7 & 3 & 98.98 & 98.98 & 98.99 & 99.14 & 99.15 & 99.15 \\
\hline 7 & 5 & 98.39 & 98.43 & 98.42 & 98.40 & 98.41 & 98.41 \\
\hline 7 & 7 & 97.74 & 97.82 & 97.83 & 97.10 & 97.13 & 97.13 \\
\hline 7 & 10 & 96.72 & 96.83 & 96.87 & 93.84 & 93.90 & 93.91 \\
\hline 7 & 15 & 95.30 & 95.68 & 95.75 & 83.36 & 83.44 & 83.46 \\
\hline 10 & 3 & 98.47 & 98.47 & 98.47 & 99.11 & 99.12 & 99.12 \\
\hline 10 & 5 & 97.82 & 97.85 & 97.87 & 98.27 & 98.29 & 98.30 \\
\hline 10 & 7 & 96.99 & 97.07 & 97.12 & 96.98 & 97.01 & 97.01 \\
\hline 10 & 10 & 95.95 & 96.15 & 96.16 & 93.58 & 93.64 & 93.65 \\
\hline 10 & 15 & 94.52 & 94.84 & 94.95 & 82.57 & 82.63 & 82.64 \\
\hline
\end{tabular}

Measures of efficacy using $5 \%$ imprecision level.
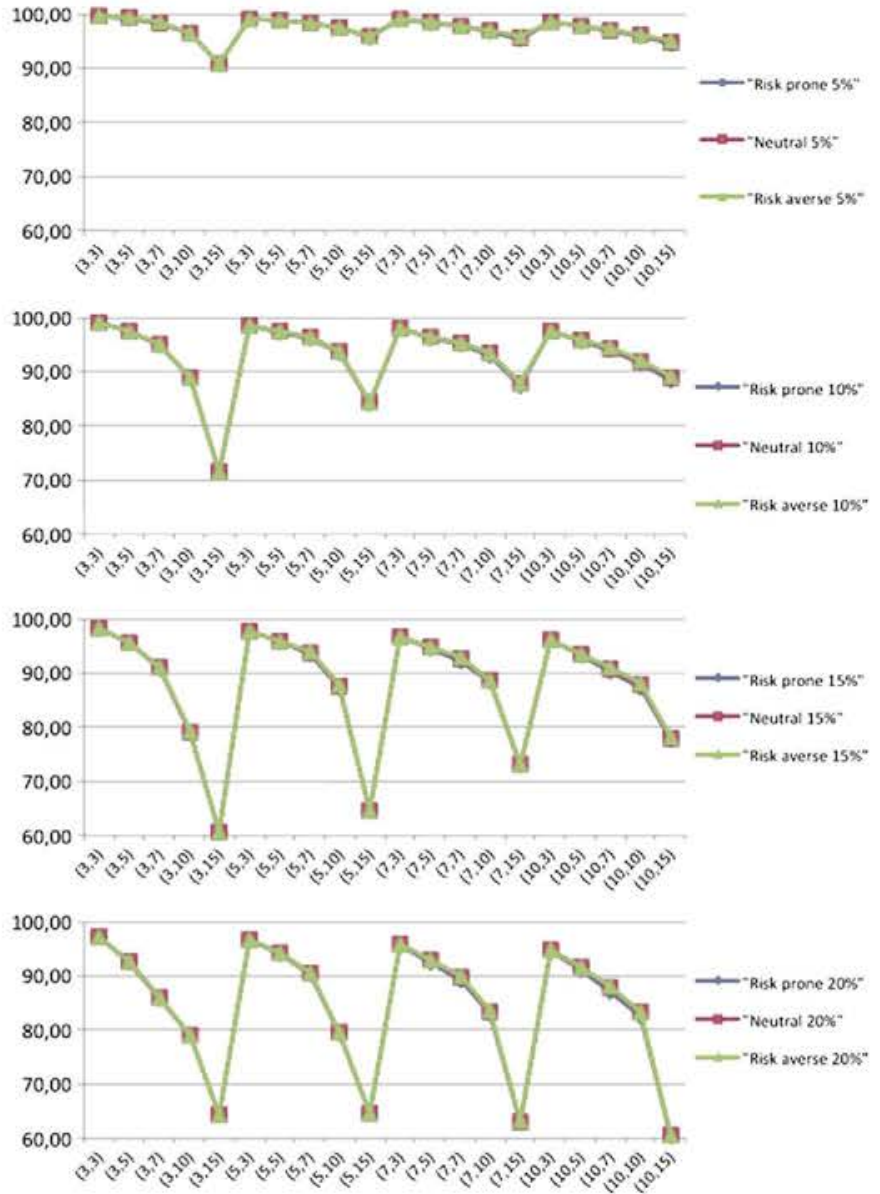

Fig. 6. Hit ratio for $5 \%, 10 \%, 15 \%$ and $20 \%$ imprecision levels.

taking into account different imprecision levels (5\%,10\%,15\% and $20 \%$ ), as shown in Fig. 5. Given a precise weight value $w_{i}$ and a imprecision level $1 \%$, the higher base of the trapezoid is $\left[w_{i}-(l / 2) / 100\right.$, 

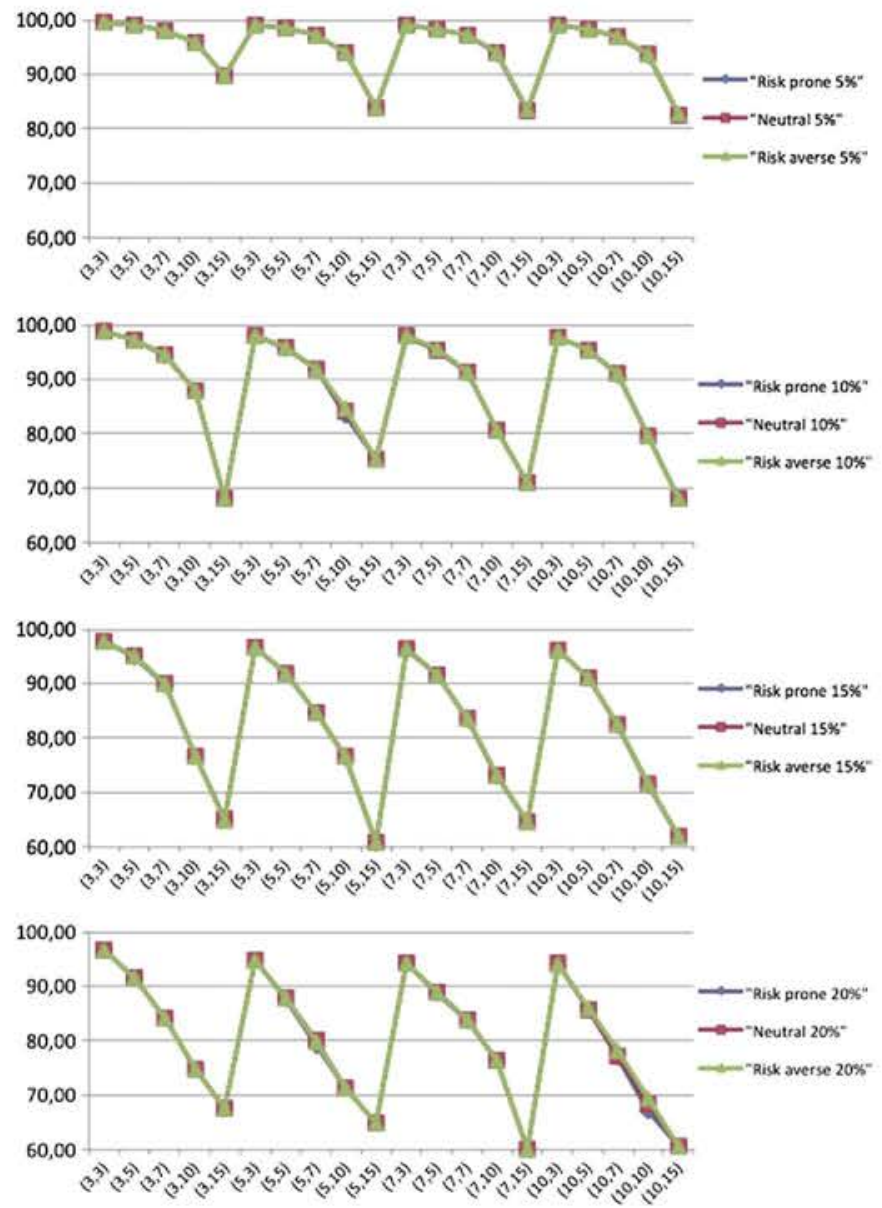

Fig. 7. Kendall's $\tau$ for $5 \%, 10 \%, 15 \%$ and $20 \%$ imprecision levels.

Table 4

Average hit ratio and Kendall's $\tau$ values.

\begin{tabular}{rll}
\hline Imprecision level (\%) & Hit ratio (\%) & Kendall's $\tau(\%)$ \\
\hline 5 & 97.32 & 94.91 \\
10 & 93.11 & 87.98 \\
15 & 87.85 & 82.35 \\
20 & 84.37 & 80.17 \\
\hline
\end{tabular}

$\left.w_{i}+(l / 2) / 100\right]$, whereas the length of the lower base is a third of the length of the higher base, both centered at $w_{i}$.

Finally, the ranking of alternatives is computed and compared with the TRUE ranking. Table 3 exhibits the average hit ratio and Kendall's $\tau$ for each of the 20 design elements with a 5\% imprecision level for the case of a risk-prone, neutral and risk-averse DM.

We can conclude that the hit ratio and the correlation coefficient are very similar for all three cases. The hit ratio is greater than $90.69 \%$ for all the design elements, whereas Kendall's $\tau$ is greater than $82.57 \%$; and both measures are decreasing regarding the number of attributes and the number of alternatives.

Figs. 6 and 7 illustrate the hit ratio and Kendall's $\tau$, respectively, for $5 \%, 10 \%, 15 \%$ and $20 \%$ imprecision levels for a riskprone, neutral and risk-averse DM. The hit ratio and the Kendall's $\tau$ are again very similar for all three cases (there is a large overlap in all cases). Both measures are decreasing regarding the number of attributes, and this decrease is more pronounced the greater the imprecision is.

The hit ratio variability (the corresponding range for a given number of alternatives) is decreasing regarding the number of alternatives for $5 \%, 10 \%$ and $15 \%$ imprecision levels. On the other hand, Kendall's $\tau$ tends to decrease with the number of alternatives. Note that when the number of alternatives is low, then each wrongly ranked alternative leads to a steep drop in Kendall's $\tau$. On the other hand, when the number of alternatives is high, then several alternatives would have to be wrongly ranked to have a big impact on Kendall's $\tau$.

Finally, Table 4 shows the average hit ratio and Kendall's $\tau$ values for the imprecision levels and a neutral DM. Both measures decrease against the level of imprecision, from $97.32 \%$ to $84.37 \%$ for the hit ratio and from $94.91 \%$ to $80.17 \%$ for Kendall's $\tau$. We consider that values for a $20 \%$ imprecision level are rather good taking into account that this is quite high imprecision. Moreover, these outcomes predictably outperforms the results output in [40], since imprecision concerning weights is represented by weight intervals rather than fuzzy numbers in [40], i.e., less meaningful information about weights is available. On the other hand, if triangular rather than trapezoidal fuzzy weights are considered regarding weights [27] then hit ratio and Kendall's $\tau$ values improve with greater imprecision.

\section{Conclusions}

Dominance-measuring methods are becoming widely used in a decision-making context with incomplete information within MAUT and have been proved to outperform other approaches, like most surrogate weighting methods or the modification of classical decision rules for application in an imprecise decision context.

In this paper, a new dominance-measuring method has been proposed, in which weights representing the relative importance of decision-making criteria are described by means of trapezoidal fuzzy numbers. The method is based on pairwise dominances and on the distances between fuzzy numbers, using the generalization of the left and right fuzzy numbers. Moreover, the cases of risk-prone, neutral and risk-averse DMs have been considered and analyzed. Monte Carlo simulation techniques have been applied to analyze the performance of the proposed method on the basis of two measures of efficacy for different imprecision levels. Hit ratio and Kendall's $\tau$ values are very similar for the different types of DMs throughout the considered imprecision levels. The method performs well, the hit ratio and Kendall's $\tau$ values being higher than $84 \%$ and $80 \%$, respectively, in the worst case ( $20 \%$ imprecision). As expected, the results are better than for similar studies accounting for weight intervals, whereas they are worse than when triangular rather than trapezoidal fuzzy weights are used, since triangular fuzzy weights provide more meaningful information about the weights.

In future research we proposed to look at other ways of representing imprecision in the problem. For instance, fuzzy numbers could be also used to represent alternative performances. Moreover, the DM could just provide an alternatives ranking for each criterion under consideration rather than performances and their utilities (assessment might be involved in real applications).

\section{Acknowledgments}

The paper was supported by Madrid Regional Government project S-2009/ESP-1594, the Spanish Ministry of Education and Science project TIN 2008-06796-C04-02 and the Spanish Ministry of Science and Innovation project MTM2011-28983-C03-03.

\section{References}

[1] Ahn BS. Extending Malakooti's model for ranking multicriteria alternatives with preference strength and partial information. IEEE Transactions on Systems, Man and Cybernetics 2003;33:281-7. 
[2] Ahn BS, Park KS. Comparing methods for multiattribute decision making with ordinal weights. Computers and Operations Research 2008;35:1660-70.

[3] Bárdossy A, Duckstein L. Fuzzy rule-based modeling with applications to geophysical, biological and engineering systems. Boca Raton: CRC Press; 1995.

[4] Barron F, Barrett B. Decision quality using ranked attribute weights. Management Science 1996;42:1515-23.

[5] Brans JP, Mareschal B. Promethee methods. In: Figueira J, Greco S, Ehrgott M, editors. Multiple criteria decision analysis. State of the art survey. New York: Springer; 2005. p. 163-95.

[6] Buckley J. Fuzzy hierarchical analysis. Fuzzy Sets and Systems 1985;17: 233-47.

[7] Bufardi A, Gheorghe R, Xirouchakis P. Fuzzy outranking methods: recent developments. Fuzzy multi-criteria decision making. Theory and applications with recent developments. Berlin/Heidelberg: Springer; 2008. pp. 119-57.

[8] Carlsson C, Fullér R. Fuzzy reasoning in decision making and optimization. Studies in fuzziness and soft computing series. Berlin/Heidelberg: Springer; 2001.

[9] Chameau JL, Santamarina JC. Membership functions I: comparing methods of measurement. International Journal of Approximate Reasoning 1987;1: 287-301.

[10] Chameau JL, Santamarina JC. Membership functions II: trends in fuzziness and implications. International Journal of Approximate Reasoning $1987 ; 1: 310-7$.

[11] Chamodrakas I, Martakos D. A utility-based fuzzy TOPSIS method for energy efficient network selection in heterogeneous wireless networks. Applied Soft Computing 2011;11:3734-43.

[12] Chan FTS, Kumar N. Global supplier development considering risk factors using fuzzy extended AHP-based approach. Omega 2007;35:417-31.

[13] Chan FTS, Kumar N, Choy KL. Decision making approach for the distribution centre location problem in a supply chain network using the fuzzy-based hierarchical concept. Proceedings of The Institution of Mechanical Engineers Part B: Journal of Engineering Manufacture 2007;221:725-39.

[14] Chang DY. Extent analysis and synthetic decision. Optimization techniques and applications, vol. 1. Singapore: World Scientific; 1992. p. 352

[15] Chen LH, Lu HW. An approximate approach for ranking fuzzy numbers based on left and right dominance. Computers and Mathematics with Applications 2001;41:1589-602.

[16] Chen SJ, Hwang CL. Fuzzy multiple attribute decision making: methods and applications. New York: Springer-Verlag; 1992.

[17] Chiou H-K, Tzeng G-H, Cheng D-C. Evaluating sustainable fishing development strategies using fuzzy MCDM approach. Omega 2005;35(3):312-25.

[18] Clir GJ, Yuan B. Fuzzy sets and fuzzy logic. Theory and application. Englewood Cliffs: Prentice-Hall; 1995.

[19] Dubois D, Prade H. Fuzzy sets and systems: theory and applications. New York: Academic Press; 1980.

[20] Edwards W, Barron FH. SMART and SMARTER: improved simple methods for multiattribute utility measurement. Behaviour and Human Decision Processes 1994;60:306-25.

[21] Eum Y, Park KS, Kim H. Establishing dominance and potential optimality in multi-criteria analysis with imprecise weights and values. Computers and Operations Research 2001;28:397-409.

[22] Farquhar PH. Utility assessment methods. Management Science 1984;30: $1283-300$.

[23] Figueira J, Mousseau V, Roy B. Electre methods. In: Figueira J, Greco S, Ehrgot $M$, editors. Multiple criteria decision analysis. State of the art survey. New York: Springer; 2005. p. 133-62.

[24] Geldermann J, Rentz O. Integrated technique assessment with imprecise information as a support for the identification of best available techniques (BAT). OR-Spektrum 2001;23:137-57.

[25] Harker PT. Alternative modes of questioning in the analytic hierarchy process. Mathematical Modeling 1987;9:353-60.

[26] Hatami-Marini A, Tavana M. An extension to Electre I method for group decision-making under a fuzzy environment. Omega 2011;39:373-86.

[27] Jiménez A, Mateos A, Sabio P. Ranking alternatives on the basis of the intensity of dominance and fuzzy logic within MAUT. Keynotes and extended abstracts of the operations research society conference OR52 2010., p. 8.

[28] Jiménez A, Ríos-Insua S, Mateos A. A generic multi-attribute analysis system. Computers and Operations Research 2006;33:1081-101.

[29] Jiménez A, Ríos-Insua S, Mateos A. Missing consequences in multi-attribute utility theory. Omega 2009;37:395-410.

[30] Kahraman C. Fuzzy multi-criteria decision making. Theory and applications with recent developments. In: Optimization and its application, vol. 16. New York: Springer-Verlag; 2008.

[31] Keeney RL, Raiffa H. Decision with multiple objectives: preferences and value-tradeoffs. New York: Wiley; 1976.

[32] Kirkwood CW, Corner JL. The effectiveness of partial information about attribute weights for ranking alternatives in multiattribute decision making. Organizational Behavior and Human Decision Processes 1993;54:456-76.

[33] Kwiesielewicz M, van Uden E. Ranking decision variants by subjective paired comparisons in cases with incomplete data. Lecture notes in computer science, vol. 2669, Computational science and its applications (ICCSA 2003). Berlin: Springer; 2003. pp. 208-15.

[34] Lahdelma R, Salminen P. SMAA-2: stochastic multicriteria acceptability analysis for group decision making. Operations Research 2001;49:444-54.

[35] Lahdelma R, Makkonen S, Salminen P. Two ways to handle dependent uncertainties in multi-criteria decision problems. Omega 2009;37:79-92.
[36] Lahdelma R, Miettinen K, Salminen P. Ordinal criteria in stochastic multicriteria acceptability analysis (SMAA). European Journal of Operational Research 2003;147(1):117-27.

[37] Lee K, Park KS, Kim H. Dominance, potential optimality, imprecise information, and hierarchical structure in multi-criteria analysis. Computers and Operations Research 2002;29:1267-81.

[38] Malakooti B. Ranking and screening multiple criteria alternatives with partial information and use of ordinal and cardinal strength of preferences. IEEE Transactions on Systems, Man and Cybernetics 2000;30:787-801.

[39] Mateos A, Jiménez A, Blanco JF. Ranking methods based on dominance measures accounting for imprecision. In: Rossi F, Tsoukis A, editors. Algorithmic decision theory. Lecture notes in artificial intelligence, vol. 5783. Berlin: Springer; 2009. p. 328-39.

[40] Mateos A, Jiménez A, Blanco JF. Dominance measuring method performance under incomplete information about weights. Journal of Multi-Criteria Decision Analysis. http://dx.doi.org/10.1002/mcda.1467. in press.

[41] Mateos A, Jiménez A, Blanco JF. Two MCDM ranking methods based on preference intensity measures: computational study. Group Decision and Negotiation; submitted for publication.

[42] Mateos A, Ríos-Insua S, Jiménez A. Dominance, potential optimality and alternative ranking in imprecise decision making. Journal of the Operational Research Society 2007;58:326-36.

[43] Mesiar A. Fuzzy set approach to the utility, preference relations, and aggregation operators. European Journal of Operational Research $2007 ; 176$ : 414-22.

[44] Papadopoulos A, Karagiannidis A. Application of the multicriteria analysis method Electre III for the optimization of decentralised energy systems. Omega 2008;36:766-76.

[45] Park K. Mathematical programming models for characterizing dominance and potential optimality when multicriteria alternative values and weights are simultaneously incomplete. IEEE Transactions on Systems, Man and Cybernetics 2004;34:601-14.

[46] Puerto J, Marmol AM, Monroy L, Fernández FR. Decision criteria with partial information. International Transactions in Operational Research 2000;7:51-65.

[47] Quattrone A, Vitetta A. Random and fuzzy utility models for road route choice. Transportation Research Part E: Logistics and Transportation Review 2011;47:1126-39.

[48] Raiffa $H$. The art and science of negotiation. California: Harvard University Press; 1982

[49] Ríos Insua D. Sensitivity analysis in multi-objective decision making. New York: Springer; 1990.

[50] Ríos D, French S. A framework for sensitivity analysis in discrete multi-objective decision-making. European Journal of Operational Research 1991;54: $176-90$.

[51] Ríos-Insua S, Gallego E, Jiménez A, Mateos A. A multi-attribute decision support system for selecting environmental intervention strategies for radionuclide contaminated freshwater ecosystems. Ecological Modeling 2006;196: 195-208.

[52] Saaty TL. The analytic hierarchy process. New York: McGraw-Hill Book Co; 1980.

[53] Saaty TL, Vargas L. Uncertainty and rank order in the analytic hierarchy process. European Journal of Operational Research 1987;32:107-17.

[54] Sage A, White CC. ARIADNE: a knowledge-based interactive system for planning and decision support. IEEE Transactions on Systems, Man and Cybernetics 1984:14:35-47.

[55] Salo AA, Hämäläinen RP. Preference programming through approximate ratio comparisons. European Journal of Operations Research 1995;82:458-75.

[56] Salo AA, Hämäläinen RP. Preference ratio in multiattribute evaluation (PRIME) - elicitation and decision procedures under incomplete information. IEEE Transactions on Systems, Man and Cybernetics 2001;31:533-45.

[57] Sarabando P, Dias LC. Simple procedures of choice in multicriteria problems without precise information about the alternatives values. Computers and Operations Research 2009;37:2239-47

[58] Stewart TJ. A critical survey on the status of multiple criteria decision making: theory and practice. Omega 1992;20:569-86.

[59] Stewart TJ. Robustness of additive value function method in MCDM. Journal of Multi-Criteria Decision Analysis 1996;5:301-9.

[60] Stillwell WG, Seaver DA, Edwards WA. Comparison of weight approximation techniques in multiattribute utility decision making. Organizational Behavior and Human Decision Process 1981;28:62-77.

[61] Tseng TY, Klein CM, Leonard MS. A formalism for comparing ranking procedures. In: Proceedings of 7th annual meeting of the North American fuzzy information processing society; 1988. p. 231-5.

[62] Tran L, Duckstein L. Comparison of fuzzy numbers using a fuzzy number measure. Fuzzy Sets and Systems 2002;130:331-41.

[63] Turksen IB. Measurement of membership functions and their acquisition. Fuzzy Sets and Systems 1991;40:5-38.

[64] Van laarhoven PJM, Pedrycz W. A fuzzy extension of saaty's priority theory. Fuzzy Sets and Systems 1983;11:229-41.

[65] Winkler RL, Hays WL. Statistics: probability, inference and decision. New York: Holt, Rinehart \& Winston; 1985

[66] Yeh C-H, Deng H. A practical approach to fuzzy utilities comparison in fuzzy multicriteria analysis. International Journal of Approximate Reasoning 2004;35:179-94

[67] Yuan Y. Criteria for evaluating fuzzy ranking methods. Fuzzy Sets and Systems 1991;44:139-57. 